\title{
Corela
}

Cognition, représentation, langage

HS-28 | 2019

Les affordances langagières : textualité numérique, matérialité discursive

\section{De l'affordance injonctive à la créativité discursive : l'exemple du ticker numérique}

\section{Catherine Ruchon}

\section{CpenEdition}

Journals

Édition électronique

URL : http://journals.openedition.org/corela/8457

DOI : $10.4000 /$ corela. 8457

ISSN : 1638-573X

Éditeur

Cercle linguistique du Centre et de I'Ouest - CerLICO

Référence électronique

Catherine Ruchon, « De l'affordance injonctive à la créativité discursive : l'exemple du ticker numérique », Corela [En ligne], HS-28 | 2019, mis en ligne le 10 septembre 2019, consulté le 13 septembre 2019. URL : http://journals.openedition.org/corela/8457 ; DOI : 10.4000/corela.8457

Ce document a été généré automatiquement le 13 septembre 2019.

\section{cc) (†)(ด)}

Corela - cognition, représentation, langage est mis à disposition selon les termes de la licence Creative Commons Attribution - Pas d'Utilisation Commerciale - Partage dans les Mêmes Conditions 4.0 International. 


\title{
De l'affordance injonctive à la créativité discursive : l'exemple du ticker numérique
}

\author{
Catherine Ruchon
}

\section{Introduction}

1 Théorisée par Gibson $(1938,1966,1977,1979)$ et largement reprise en sciences sociales (Bradner 2001; Kaufmann \& Clément 2007; Franchak, Van Der Zalm \& Adolph 2010; Paveau 2012 entre autres), la notion d'affordance est une des clefs permettant de sortir d'une pensée binaire et anthropocentrée. Plus spécifiquement, il s'agit dans cet article de questionner les affordances, autrement dit les «signes actanciels» (Uexküll 2010 [1956-1934]), les «valences » ou le " caractère de demande » (Lewin 1926, Werner 1926) d'objets numériques iconotextuels (Nerlich 1990) tels que le ticker, sorte d'échelle temporelle très utilisée par les internautes sur les forums associés à des événements (future naissance, projet de mariage, etc.). On peut supposer que si les objets incitent à l'action, ainsi que la théorie des affordances le stipule, la liberté d'agir est partiellement contrainte dès lors qu'il s'agit d'artefacts et d'objets numériques. Forme d'architexte (Genette 1979, Jeanneret et Souchier 2005), le ticker propose un modèle iconique et langagier qui simultanément incite à l'action tout en limitant ce champ d'action. La diversité d'affordances (prévisibles et imprévisibles) ne se réduit pas aux potentialités intrinsèques à l'objet mais elle est aussi liée à l'expérience et aux besoins de l'usager. De ces derniers dépend la possibilité de dépasser le niveau injonctif de l'affordance. S'affranchissant des affordances prévues par les concepteurs et de leurs intentionnalités, les usagers "détournent " les fonctionnalités d'objets numériques en conservant le design initial mais en modifiant les éléments textuels qui l'accompagnent.

Après avoir dessiné les contours historiques de la notion d'affordance, je montrerai que l'on peut distinguer des affordances primaires, auxquelles s'associe une réponse spontanée et unique, et des affordances secondaires, qui nécessitent plusieurs gestes- 
réponses et peuvent autoriser une certaine créativité. L'exemple du ticker numérique permet ainsi de souligner comment l'internaute détourne les usages qui sont attendus.

\section{Parcours historique de la notion}

3 Le nom de Gibson laisse souvent dans l'ombre celui des autres chercheurs qui ont contribué à élaborer la théorie des affordances. Celle-ci est aujourd'hui convoquée dans différents champs, l'enseignement et surtout le design, domaine où l'on cherche tout particulièrement à anticiper les affordances d'un produit.

\subsection{Des gestaltistes à Gibson}

4 Heinz Werner et Jakob von Uexkül, qui enseignèrent tous deux à l'Université de Hambourg, sont deux précurseurs de la notion d'affordance. Selon Werner, psychologue austro-américain du développement de l'enfant, les objets, en devenant des «objetssignaux », provoquent des comportements types. En 1926, il écrit :

"Jusqu'à ce que notre activité soit limitée à une situation totalement concrète, familière, les objets semblent guider, conduire, notre action en vertu de leurs qualités de signal. Lorsque nous sommes fatigués, une chaise vide nous "invite" à nous asseoir. Dans une telle situation la chaise montre (...) un "caractère de demande", ou une "valence" qui nous encourage à nous asseoir. (1926/1948, 61; Trad. A.)» (Werner 1926, cité par Niveleau 2006 : 161)

Werner montra qu'un hochet ne prend une signification que lorsque le tout jeune enfant comprend qu'il peut le presser entre ses gencives. La relation entre l'expérience et la fonction, sur laquelle je reviendrai, est déterminante.

6 À sa suite, Jakob von Uexküll, naturaliste et biologiste allemand qui a travaillé sur le milieu (l'umwelt) de l'animal, s'approche de très près du concept d'affordance sans encore le nommer ainsi. Tout d'abord, il oppose les « outils» (les moyens pour agir, par exemple les machines d'usine qui permettent de transformer les produits, les voitures, etc.) et les «perceptils» (les machines pour percevoir comme le télescope, la lunette, la radio) (Uexküll 2010 [1934] : 26). Plus précisément, Uexküll parle de «signe actanciel» et de «tonalité » («tonalité de s'asseoir »), et plus explicitement de "tonalité actancielle» (Uexküll 2010 [1934]) : 112). On s'approche des affordances, et nous ne sommes que dans les années 1930.

7 C'est à Kurt Lewin (1926: 28) que l'on doit le terme d'affordance (Aufforderungscharakter) mais l'expression allemande fut traduite par le mot anglais « valence » sur la proposition du psychologue E. C. Tolman (1932) qui avait développé un concept similaire (« demandevalue »). Cette proposition fut acceptée par Lewin (Niveleau $2006: 166)$. La valence est l'attraction ou la répulsion suscitée par les objets (Lewin 1935 [1926] : 50). Cela implique de prendre en considération tout l'environnement du sujet, ce que Lewin appelle «l'espace de vie psychologique ».

8 La notion sera aussi travaillée par le collègue de Lewin, Köhler $(1929,1938)$, qui parle de « réquisition »: au niveau phénoménal, l'objet, et plus largement l'environnement physique, interpelle, pouvant susciter rejet ou acceptation. Köhler veut élaborer une 
théorie de l'expérience qui associe dans la perception les faits et les valeurs (esthétiques, émotionnelles, morales).

L'idée d'intégrer ainsi les valeurs, les émotions et tout ce qui relève de l'expressivité dans les données de l'expérience fera son chemin dans le courant gestaltiste, notamment chez le psychologue Kurt Koffka (1935). Cela implique de considérer la conation en relation avec l'environnement psychologique.

10 Un autre nom a fortement marqué l'histoire, celui du psychologue James J. Gibson, spécialiste de la perception visuelle. C'est l'homme de la rupture, celui qui prend ses distances avec la théorie gestaltiste. C'est aussi celui qui popularise le mot affordance, et à qui l'on attribue souvent la paternité du concept. Gibson s'est beaucoup appuyé sur les travaux de ses prédécesseurs, et notamment sur la notion de valence :

«Par valences, positive ou négative, nous parlons de la signification des objets en vertu de laquelle nous nous approchons de certains et nous nous éloignons d'autres ». (J. J. Gibson \& Crooks, b, 454-455 / Traduction de l'auteur (Trad. A.). (Gibson \& Crooks 1938, cité par Niveleau $2006: 160$ )

11 Mais contrairement à Köhler pour qui la valence est une propriété psychologique, Gibson considère l'affordance comme une propriété écologique, se démarquant ainsi de la tradition gestaltiste. Chez Gibson, la valence devenue affordance ne conserve que la dimension motrice (Niveleau 2006: 179). Le théoricien évacue ainsi ce qui concerne la dimension expressive et les valeurs présentes dans la théorie de Köhler, mais aussi chez Koffka, et avant eux chez Werner pour qui les valeurs émotionnelles (d'un visage, mais aussi, dans un mode de cognition plus général, d'un paysage ou de tout objet) sont immédiatement perçus (Werner 1957 [1926] : 69). A la suite de Gibson, les psychologues de la perception ne prendront pas en compte l'expressivité (Niveleau 2006 : 179).

Spécialiste de la perception visuelle, comme l'était Werner, Gibson élabore une première définition de l'affordance dans son ouvrage de 1966, The Ecological Approach to Visual Perception ${ }^{7}$ :

«Quand les propriétés constantes des objets constants sont perçues (la forme, la taille, la couleur, la texture, la composition, le mouvement, l'animation et la position relative par rapport aux autres objets), l'observateur peut continuer de détecter leurs affordances. J'ai inventé ce mot comme un substitut des valeurs, terme qui porte un lourd fardeau de signification philosophique. Je veux simplement dire que les choses "fournissent" [furnish], de façon positive ou négative. Tout ce qu'elles "procurent" [afford], après tout, dépend de leurs propriétés. (Gibson, 1966, 285 /Trad. A. ; nos italiques). » (Gibson 1966, cité par Niveleau 2006 : 179)

13 Dans cette première définition, ainsi que le note Niveleau (2006:180), Gibson dérive les affordances des propriétés de l'objet, tandis que dans une définition postérieure, le psychologue distingue nettement propriétés et affordances en affirmant que «ce que nous percevons, quand nous regardons des objets, sont leurs affordances, pas leurs qualités» (Gibson 1979: 134). Ainsi, les affordances sont perçues directement (Gibson 1979 : 139-140). C'était aussi ce qu'affirmait le gestaltiste Kurt Koffka, qui fut le collègue de Gibson à la fin des années 1920. Les choses prendraient leur sens en fonction d'une perception immédiate : «[...] a fruit says "Eat me"; waters says "Drink me" [...]» (Koffka, 1935 : 7). Mais selon Koffka, ces significations découlent de l'expérience, point sur lequel je reviens plus bas, et de la nécessité (j'identifie la fonction d'un objet parce que j'en ai 
l'utilité à un moment $\mathrm{t}$ ). Il en est de même chez Lewin, pour qui la signification de l'objet varie selon les besoins de l'individu. Pour Gibson au contraire, l'affordance est constitutive de l'objet, et n'est donc pas soumise aux besoins du sujet. Ainsi Koffka développe une approche phénoménologique là où Gibson défend une vision écologique et systémique.

\subsection{L'anticipation d'affordance : un exemple dans le domaine du design}

14 La notion d'affordance a été exploitée concrètement dans deux grands domaines, l'apprentissage (par exemple Gaisch 2015, Leńko-Szymańska \& Boulton 2015) et, ce qui nous intéresse davantage, dans le design. C'est en effet un concept-clé pour élaborer des produits intuitifs et faciles à utiliser. Donald Norman a introduit et popularisé la notion dans le design à la fin des années 1980, notamment dans la relation « homme-machine » (Norman 1988).

15 A sa suite, la notion a été explorée par des théoriciens du design comme Viktor Kaptelinin (2014), et plus récemment, par le designer Benjamin Loyauté (2016, 2017). Son travail montre bien comment un concepteur peut anticiper l'affordance d'un produit. A la fois auteur, vidéaste, désigner et anthropologue, Loyauté a conçu un objet à partir d'un objectif précis. Il s'agit d'un bonbon, ou plutôt d'une sucrerie narrative. Appelée Louloupti, cette friandise évoque l'histoire de la Syrie par sa forme de "petit fétiche", par son goût (celui d'un bonbon syrien existant), par son parfum à la rose (ce qui le légitimise comme sucrerie arabe). Tout cela confère au louloupti une grande crédibilité comme objet fictionnel support de mémoire. Cette évocation du passé vise à faire prendre conscience de la situation actuelle en Syrie. A la façon de Latour (1994), Loyauté considère les objets comme des «agents d'informations et des actants» (Loyauté 2017: 39), des «objets-mots» dotés d'une force perlocutoire: «Je n'ai jamais réduit un objet à la servitude de son usage » (Loyauté 2017 : 38). Le bonbon n'a pas été créé seulement pour éveiller la gourmandise, il est aussi incitation à la mémoire et à l'action. D'une certaine manière, Loyauté anticipe des affordances indirectes possibles à partir d'un bonbon dans le contexte syrien. Cela pose la question de la réception. Peut-on maîtriser aussi facilement l'enchaînement affordance / action produite? Norman, qui explore les potentialités d'action perceptibles par l'utilisateur d'un programme, précise sur son blog la notion d' "affordance perçue », rappelant que ce qui prime pour le designer, c'est ce que l'utilisateur perçoit et non ce qui est vrai :

«I suspect that none of us know all the affordances of even everyday objects.

[...] in design, we care much more about what the user perceives than what is actually true. What the designer cares about is whether the user perceives that some action is possible (or in the case of perceived non-affordances, not possible).

[...] The real question is about the perceived affordance: Does the user perceive that clicking on that location is a meaningful, useful action to perform? » (Norman 2004, en ligne)

16 Ce questionnement de Norman rejoint en partie ma problématique, qui se place sur le plan des praxis: comment se combinent perception, émotion et expérience dans l'exercice du libre agir de l'usager face à l'objet numérique ? Les routines installées le sont 
toujours de manière provisoire, elles ne constituent qu'un « fonds précaire » qui stimule une " activité créatrice » (Leroi-Gourhan 1945 : 426). Au-delà des affordances prévues par les concepteurs et de leurs intentionnalités, dans des situations spécifiques, les usagers « détournent » les fonctionnalités d'objets numériques en conservant le design initial mais en modifiant les éléments textuels qui l'accompagnent, créant ainsi une nouvelle catégorie, plus invisible, de produsage (Bruns 2008). Les internautes parviennent ainsi à créer une rupture de cadre (Goffman 1974), en modifiant notamment les affects qui lui conventionnellement associés.

\section{Les affordances, entre expressivité, expérience et besoin}

17 Mon objet d'étude, le ticker numérique, m’a amenée à relier les différentes approches théoriques, entre position gestaltiste et conception écologique.

\subsection{Une signification holistique}

18 En accord avec les précurseurs de la notion, je privilégie l'idée de la primauté du milieu (l'« umwelt» de Uexkhüll), suivant aussi en cela l'approche écologique gibsonnienne. Chez Uexkhüll en effet, le chêne n'a pas les mêmes tonalités (ou affordances, selon le mot moderne) pour le renard ou pour l'écureuil. Les racines de l'arbre ont pour le renard la tonalité d'un abri, tandis que les ramifications ont pour l'écureuil une tonalité d'ascension (Uexkhüll 2010 [1934] : 158). Chez Gibson, l'affordance «envoi de lettres » d'une boîte aux lettres ne peut exister que dans un environnement où la boîte aux lettres a déjà cette fonction (Gibson 1979 : 139).

19 Mais cela n'apparaît pas suffisant pour analyser ce que font les internautes lorsqu'ils détournent les affordances d'un ticker. J'ai voulu approfondir la dimension motrice qui est au cœur de la théorie de Gibson, en étudiant comment les usagers peuvent dépasser les significations directement perçues en complétant de façon objective les propriétés de l'objet par l'attribution d'une nouvelle fonction, comme la transformation d'un ticker de vacance en ticker de deuil (cf. infra). Selon moi, la perception dépend de l'investissement dynamique dans l'environnement, mais aussi des valeurs émotionnelles de l'objet et des propriétés imaginaires qu'on lui prête.

20 Je m'appuie tout particulièrement sur une conception de la signification telle que l'envisage Werner : la signification peut être élaborée à partir d'éléments imaginaires. Tel l'enfant lorsqu'il attribue à un brin de paille la signification " poupée » en attribuant des propriétés imaginaires à l'objet (Werner 1957 [1926] : 65). Je retiens de la démarche gestaltiste l'idée de la valeur et de l'expressivité (l'objet "ticker» étant porteur d'émotions directement perceptibles), et d'une vie intérieure de l'objet qui forme son «caractère physionomique» (Werner 1957 [1926]: 69). L'objet ne se contente pas d'envoyer des informations, il joue un rôle d'interpellation en fonction de l'état émotionnel du sujet et de ses besoins (ponctuels). Il nous incite à agir selon un effet d'attirance ou de répulsion (Gibson \& Crooks 1938: 454-455). Plus encore, les informations émises sont interprétées différemment selon la situation. C'est ainsi que certains objets peuvent être détournés de leur usage primitif, celui pour lequel ils ont été pensés et conçus. Ainsi, dans un second mouvement, en fonction des émotions et des besoins qui l'animent, le sujet peut éventuellement modifier les affordances «prévisibles» de l'objet. On voit que la signification n'est pas le produit direct des 
propriétés objectives et matérielles de l'objet mais d'un faisceau d'éléments (dont les émotions) contribuant aux affordances de l'objet.

Dans ce qui suit, je vais donc explorer l'idée des potentialités affordantes imprévisibles en cherchant à établir les relations entre les propriétés de l'objet numérique avec les valeurs émotionnelles, le besoin et l'expérience du sujet percevant.

\subsection{L'expérience comme condition praxique de l'affordance}

Dans son ouvrage Milieu animal et milieu humain, Uexküll écrit : "Comment faisons-nous pour voir dans la chaise le s'asseoir, dans la tasse le boire, dans l'échelle le grimper [...] ?» (Uexküll 2010 [1934] : 109). Le naturaliste donne l'exemple du chien à qui l'on ordonne de s'asseoir sur une chaise. Puis on renouvelle l'ordre mais sans la chaise : le chien saute alors sur tous les objets avec lesquels il pouvait effectuer la même performance « s'asseoir ». Uexküll explique que ces objets (caisse, étagères...) avaient tous la « tonalité de s'asseoir » (Uexküll 2010 [1934] : 108). On parlerait aujourd'hui d'affordances. Mais qu'est-ce qui rend si évidente cette tonalité ?

Pour mieux l'expliquer, Uexküll donne un autre exemple, celui d'un Africain qui ne connaissait pas les objets d'usage européen. Face à une échelle qu'Uexküll l'enjoint à gravir, il s'interroge "Comment faut-il faire ? Je ne vois que des barres et des trous. " (Uexküll 2010 [1934] : 109). Effectivement, les différents objets constitués de barres et de trous (grille de jardin, échelle, charriot de boulangerie, étagères, voire oculus, etc.) n'ont pas tous le même usage.

Ainsi que l'exprime Morgagni (2011) dans son article « Repenser la notion d'affordance dans ses dynamiques sémiotiques ", les affordances sont fortement liées à la dimension sociale et culturelle. Si on n'a jamais rencontré ces objets, sans mode d'emploi, peuventils afforder quelque chose ? Est-ce que l'on pense à grimper lorsque l'on voit une grille? Et pourtant, il s'agit bien de "trous et de barres »! Mais si l'on doit échapper à un danger, et que l'on connaisse le principe de l'échelle, tout outil à barreaux (comme les barreaux d'une grille) pourrait inciter à grimper. Ainsi que l'écrit Saadi Lahlou à propos des attracteurs cognitifs :

« Il ne faut donc pas croire que les sujets répondent à des attracteurs de manière mécanique, comme des animaux conditionnés à un stimulus : ils construisent et modifient activement, et en général à plusieurs, ces attracteurs; et ils en sont conscients. » (Lahlou 2000: 90, cité par Paveau 2018, en ligne)

On peut donc dire que l'expérience préexiste à l'affordance. L'affordance des objets dépend des modèles : nous avons vu nos parents boire dans un verre, une tasse, un bol, une bouteille, une assiette creuse. Nous en avons appris l'usage et l'habitude s'est instaurée. La signification du signe, au sens de Peirce, se stabilise. Dans ses travaux sur l'interprétation (immédiate, dynamique, finale), Charles S. Peirce montre en effet que si le processus sémiotique est théoriquement illimité ${ }^{1}$, il peut être bloqué par l'habitude (Poche 2009: 558-559).

De l'habitude (le figement du renvoi d'un signe à d'autres signes) peut resurgir la nouveauté. Les internautes s'appuient sur une connaissance préalable des tickers et d'autres signes stabilisés (comme la représentation linéaire du temps par une échelle temporelle) pour en modifier l'usage. Plus le sujet a d'expérience, plus les objets 
afforderont des choses différentes. Reprenons l'exemple de la tonalité «s'asseoir ». Je peux m'asseoir sur une chaise, un tabouret, parce que l'on m'a appris à le faire, mais je peux aussi m'asseoir sur une pierre ou un tronc d'arbre, parce que mon expérience m'a appris qu'ils avaient la densité et la dureté nécessaire pour le « s'asseoir », et que mon œil a su évaluer leur stabilité (une pierre branlante n'afforde pas le «s'asseoir»). De manière cyclique, cet usage d'abord inédit peut se stabiliser à son tour, instaurant à nouveau une routine. L'expérience est ainsi conçue dans une dynamique d'appropriation (de mise en routine) et d'innovation.

\subsection{Le besoin comme condition environnementale de l'affordance}

Une autre condition est nécessaire : pour voir dans un caillou le "s'asseoir », il faut que le sujet éprouve le besoin de s'asseoir. Ainsi que l'explique Niveleau, le caractère de demande (l'affordance) « reste alors opérant aussi longtemps qu'il existe une tension entre une personne et un environnement qui déclenche son action. Inversement, ce concept sera désactivé dès que l'environnement perdra son caractère de demande " (Niveleau 2006 : 170). La tension est liée au besoin: sa satisfaction permet de relâcher la tension (Lewin 1968 [1938] : 97).

Ainsi, la diversité d'affordances d'un objet dépend de la perception du sujet (visuelle, tactile, etc.) qui permet d'évaluer les potentialités de l'objet en fonction du besoin, de l'expérience de l'usager (à qui l'on a appris la fonctionnalité de l'objet et qui connaît les potentialités de divers objets). La combinaison des deux multiplie le coefficient affordant: si je connais le principe de l'échelle et que je vois une grille, je pourrai l'utiliser comme échelle.

Je vais maintenant présenter les deux niveaux d'affordances et montrer que lorsqu'on se situe à un niveau secondaire, le coefficient d'affordance et la liberté d'agir sont plus élevés.

\section{Affordances numériques primaires et secondaires}

Le ticker appartient à ces objets numériques qui nécessitent plusieurs gestes pour que l'affordance prévisible, celle prévue à l'origine par les concepteurs, puisse être accomplie. On peut en effet distinguer deux niveaux d'affordance, les affordances primaires, dont la perception est suivie d'une réponse immédiate, et les affordances secondaires, dont l'action qui en découle nécessite une certaine réflexion et plusieurs gestes pour son accomplissement.

\subsection{L'affordance primaire}

Le numérique propose un grand nombre d'affordances primaires qui sont à relier avec l'interface graphique que les concepteurs souhaitent rendre très intuitive. Pensons à tous les éléments de navigation: boutons, icônes, barre des tâches. Il suffit à l'usager d'exécuter un seul geste pour avoir immédiatement la réponse attendue.

En voici quelques autres exemples : 
Fig. 1 : boutons « play » et « pause » sur le site YouTube.

† Ajouter à

$\Rightarrow$ Partager $\quad \cdots$ Plus

Fig. 1bis : boutons « Ajouter à », «Partager » et « Plus » sur le site YouTube.

Abonnez vous à partir de $1 \mathrm{C}$
을

\section{f Partager (394)}

Fig.2 : site du journal Le monde. bouton play des chaînes hifi, pour pouvoir visionner la vidéo (fig. 1), et qu'il clique sur l'icône pouce en l'air ou vers le bas (fig. 1bis) pour marquer son approbation («j'aime/ j'aime pas »). Le bouton textuel « ajouter à » permet de compléter sa playlist. Le bouton "partager» donne l'accès immédiat aux icones de Twitter, FaceBook, Google+, etc. Le bouton "plus» permet différentes actions: "signaler», "transcription ", " statistiques », « ajouter des traductions ».

Sur le site du magazine Le Monde, les boutons iconotextuels « $\mathrm{f}$ partager » et « [logo oiseau] Tweeter » permettent de partager instantanément sur FaceBook ou d'accéder au site de Twitter. On peut aussi « réagir » à l'article ou « ajouter » la page à ses favoris.

Toutes ces incitations à l'action sont suivies d'une réponse immédiate : l'appréciation « j'aime » ou « j'aime pas » s'affiche, la vidéo est ajoutée à la playlist de l'usager.

Les affordances primaires ne concernent pas seulement des actions concrètes comme le visionnage d'une vidéo ou le partage d'une information, elles s'associent aussi à des actions idéologiques et mémorielles : 
Chères amies, chers amis,

Les marsouins du Pacifique - ou vaquitas - sont d'adorables mais timides mammifères marins semblables à de petits dauphins... et il n'en reste plus que 30 ! Mais si nous agissons rapidement, nous pouvons encore éviter que cette magnifique espèce ne s'éteigne à jamais.

Pris au piège de terribles "filets maillants", ces marsouins se noient et meurent. Mais le Mexique pourrait interdire ces filets, et un responsable politique de première importance a accepté de remettre notre pétition auprès des plus hautes instances gouvernementales.

Ensemble, nous pouvons faire basculer la décision en faveur des vaquitas. Montrons aux ministres concernés que s'ils proposent une interdiction totale, nous serons un million à les soutenir! Signez la pétition urgente ci-dessous en un clic et partagez tout autour de vous:

\section{Cliquez pour sauver les vaquitas}

Fig. 3 : courrier électronique d'Avaaz (organisation non gouvernementale internationale de cybermilitantisme) présentant une pétition en faveur des vaquitas (marsouins du Pacifique) (2017)

Ci-dessus, si l'internaute qui a reçu ce mail d'Avaaz clique sur le lien « Signez pour sauver les vaquitas ", le résultat est instantané: il est aussitôt remercié pour son geste. L'instantanéité est d'ailleurs soulignée par le commentaire du cotexte de gauche, en un clic («Signez la pétition en un clic et partagez tout autour de vous »). Ces injonctions permettent un engagement immédiat que n'a pas forcément recherché l'internaute.

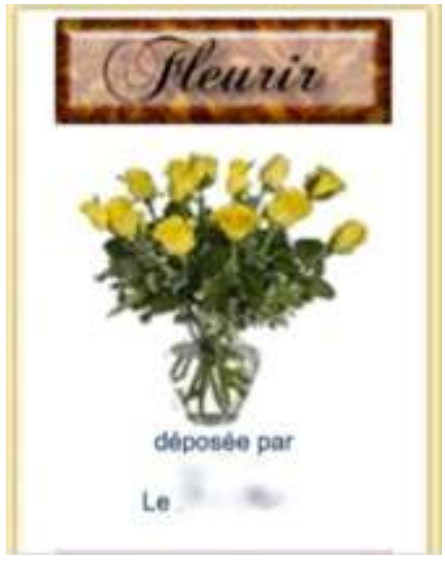

Fig. 4 : fleurs déposées sur une tombe virtuelle humaine du site « Le cimetière virtuel » en 2017. 


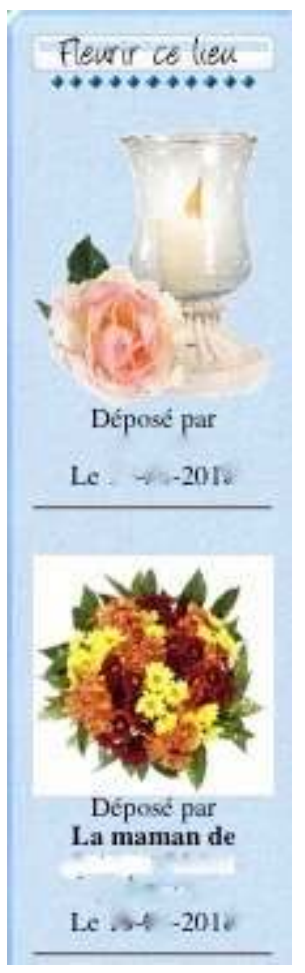

Fig. 5 : fleurs déposées sur une tombe virtuelle animale du site « Le cimetière animalier » en 2018 numériques rappellent par leur forme injonctive que derrière l'artefact il $\mathrm{y}$ a un concepteur. On voit ici des boutons qui permettent une action immédiate. Au geste répond instantanément une action à la signification non équivoque : «j'aime », «je partage ", « je m'abonne ». Mais cela ne permet pas une affordance autre que celle pour laquelle la fonctionnalité a été prévue. On remarquera d'ailleurs qu'ils fonctionnent avec le mode impératif (Signez, Abonnez vous) et l'infinitif de l'injonction (Partager, Tweeter, Fleurir). Il en est de même avec les boutons non textuels : l'image du pouce ou l'icône " play » s'interprètent comme des impératifs visuels. Je clique, j'agis, mais je ne peux interférer quoi que ce soit. Mon agir n'a qu'une réponse possible, ou inversement, l'objet n'afforde qu'un seul agir. Le ticker en revanche est un objet numérique qui laisse davantage de prise à l'initiative individuelle.

\subsection{L'affordance secondaire : l'exemple du ticker}

A ce préformatage de l'interface, à ces actions prédéterminées (ne serait-ce que la pagination d'un document Word), on peut opposer par exemple le hashtag qui pousse à aller chercher de l'information en tapant dans la barre de recherche le mot distingué par le signe dièse. C'est l'« image-perception » (Uexküll) du signe \# qui appelle à l'action. Il en est de même avec le ticker : aucun bouton « ajoutez votre ticker » n'incite à l'action. C'est 
la seule perception visuelle d'un ticker qui peut inciter l'internaute à personnaliser à son tour ses écrits numériques d'une échelle temporelle.

\subsubsection{Définition et modèles de ticker}

Selon la définition de Doctissimo: "Les tickers sont des images personnalisées dynamiques. Ils permettent d'afficher des calendriers ou des comptes à rebours. ». Le ticker est généré automatiquement après la sélection d'un fond, d'un curseur, d'un type d'événement et d'une date. La mise à jour se fait à chaque actualisation.

Cette image temporelle dynamique joue le rôle d'un déictique temporel (Ruchon 2015 : 114-117) et ancre l'énonciation dans la temporalité du récit numérique, à la fois visuellement (le curseur bouge au fur et à mesure du temps qui s'écoule) et textuellement (le texte s'ajuste automatiquement). Il s'agit donc de "modèles de textes » (Gülich \& Krafft 1997) mais aussi d'images, autrement dit d'iconotexte (Nerlich 1990).

Le ticker, élément grapho-discursif propre aux blogs, aux forums et aux mails, s'utilise essentiellement pour annoncer l'arrivée ou la naissance d'un enfant :

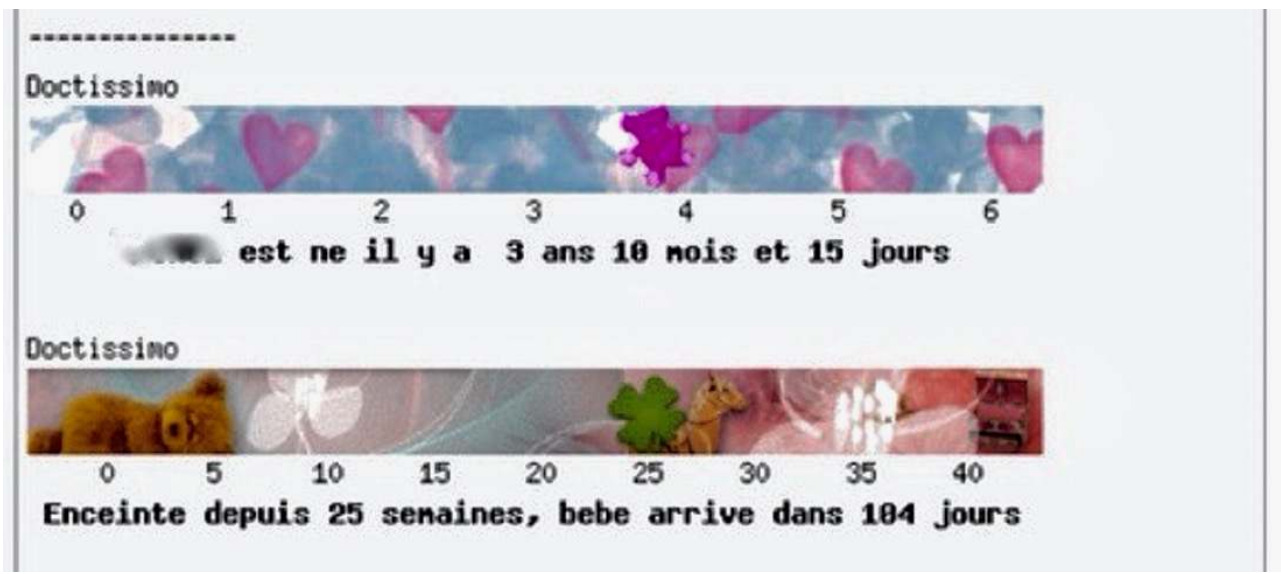

Fig. 6 : ticker de naissance du forum Grossesse sur Doctissimo.

On voit par ces deux tickers que les internautes sont fidèles au principe et réitèrent pour le second enfant.

On emploie le ticker dans d'autres contextes, comme l'arrêt du tabac. En voici un exemple où l'internaute souhaite aux autres internautes un "bon moisi à tous", moisi étant l'apocope de moisiversaire (anniversaire mensuel) :
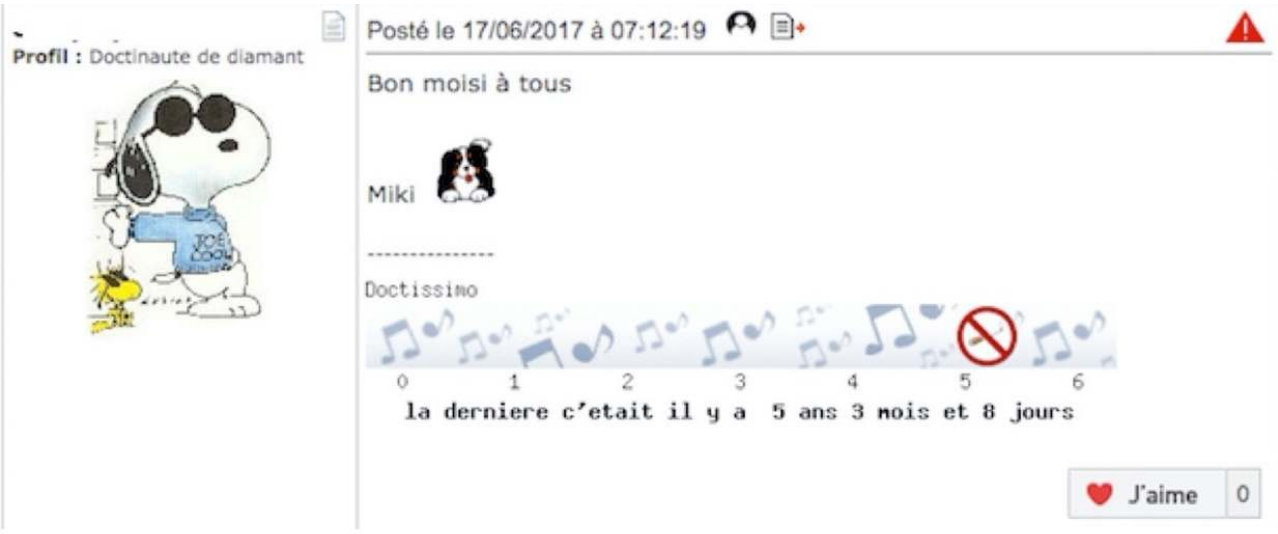

Fig. 7 : ticker d'arrêt de tabac de Doctissimo en 2017. 
L'internaute qui voit un ticker sur un forum qu'il consulte par intérêt personnel (par exemple lorsqu'un enfant est à naître ou parce qu'il souhaite s'arrêter de fumer), va être incité par imitation à ajouter à son tour un ticker à ses productions numériques. Il s'agit alors d'une affordance secondaire. Il va d'abord devoir chercher les modes d'emplois (fig. 8), soit en faisant une recherche, soit en cliquant intuitivement sur le ticker d'un usager, ce qui dans les deux cas l'amènera à cette page :

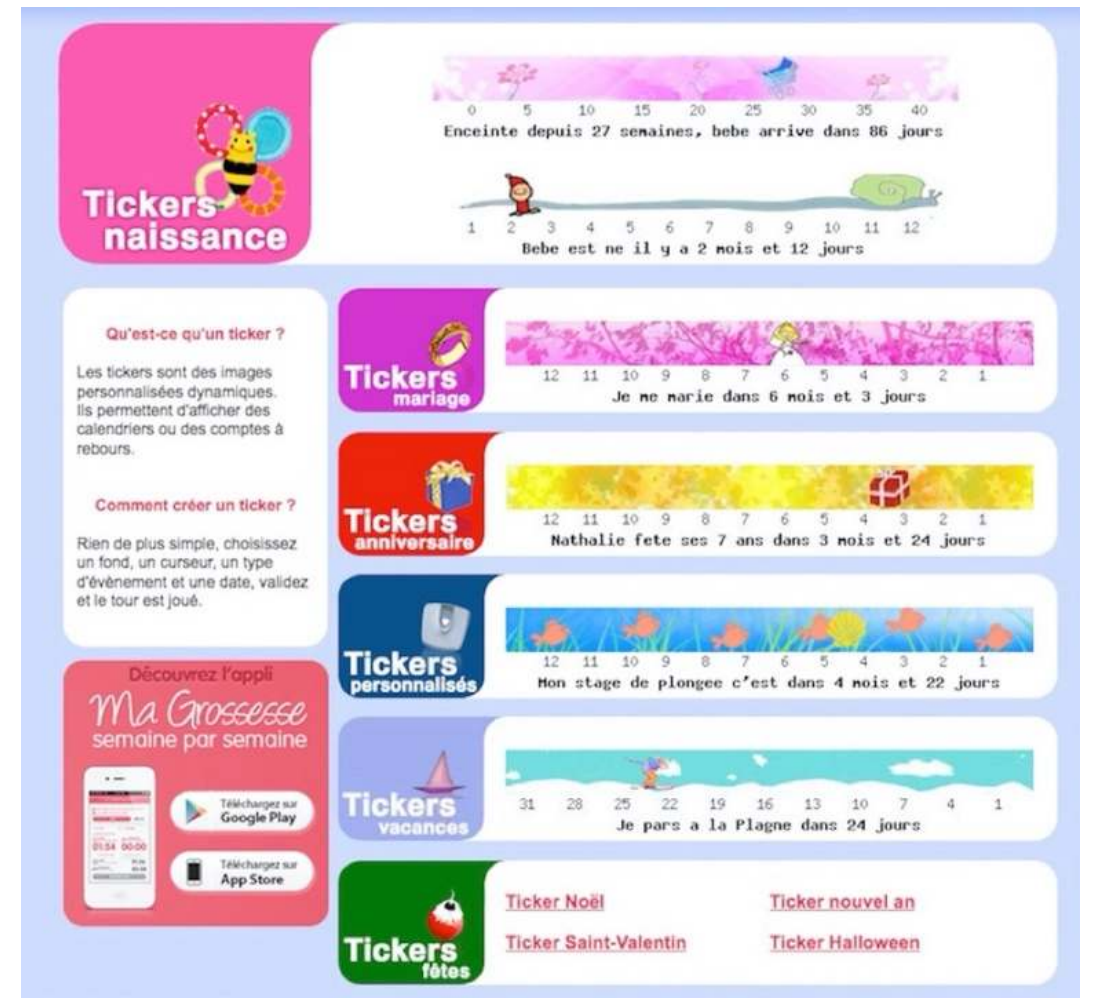

Fig. 8 : définition et mode d'emploi du ticker sur Doctissimo.

On peut donc distinguer :

- des modèles d'expérience (ce que font les autres) qui engendrent la répétition (figures 6 et 7),

- des modèles vierges d'action (fig. 8), que l'on peut qualifier d'architextes (Genette 1979, Jeanneret et Souchier 2005), qui proposent par l'exemple.

Ces modèles contribuent à poser des cadres primaires sociaux (Goffman 1974) que l'interprète-utilisateur n'aura aucune peine à décrypter. La perception de ces objets peut amener l'internaute à positionner un ticker sous sa signature, ce qu'il devrait pouvoir faire pour tout événement, mais le dispositif techno-numérique guide fortement les cadres d'usage: naissance (mis en avant par la mise en page), mariage, anniversaires, tickers personnalisés, vacances, fêtes. Les modèles d'expérience et les modèles d'usage incitent à employer le ticker pour des événements heureux, même dans le cas de tickers personnalisés (où le modèle propose l'annonce d'un stage de plongée, qui appartient à la catégorie des événements loisirs). Il en est de même sur les autres sites offrant la possibilité de tickers (lilypie, tickerclub, ticker factory, etc.). Tous proposent les mêmes tickers modélisés à partir d'événements considérés comme heureux. 


\subsubsection{De la modalisation à une nouvelle routine} intrinsèques à l'objet mais aussi de l'expérience de l'usager, et que l'expérience s'associait à l'appropriation et à l'innovation. Plus le coefficient d'expérience augmente, plus la possibilité de dépasser le niveau injonctif de l'affordance numérique augmente lui aussi et plus s'exprime la créativité des sujets. S'opère alors ce que Goffman appelle une modalisation du cadre primaire. Goffman a observé que les cadres primaires sociaux sont, plus que les cadres primaires naturels (c'est-à-dire physiques, non pilotés par l'humain), aptes à être modalisés dans un second temps (Goffman 1991 [1974] : 54). Par mode, il entend « un ensemble de conventions par lequel une activité donnée, déjà pourvue d'un sens par l'application d'un cadre primaire, se transforme en une autre activité qui prend la première pour modèle mais que les participants considèrent comme sensiblement différente » (ibid. : 52). Goffman donne l'exemple du combat qui peut être modalisé en activité ludique ou en activité sportive. Dans les exemples étudiés ici, il y a une réorientation du cadre : le ticker destiné à célébrer des événements heureux est modalisé en ticker de deuil. La pratique du ticker de deuil s'est ensuite répandue, instaurant par l'imitation une nouvelle routine. Le cadre secondaire associé au ticker de deuil est alors transformé en cadre primaire. Mais il aura fallu pour cela que les internautes parviennent à déjouer les affordances prévues par les concepteurs.

\section{Intentionnalité et détournement d'affordance}

Les artefacts sont créés avec une intentionnalité, une intention d'affordance. Nous l'avons constaté avec l'exemple du Louloupti de Benjamin Loyauté. C'est encore plus le cas pour l'objet numérique. Pourtant, en dépit du peu de liberté d'action possible, certains internautes détournent l'usage prédéfini du ticker pour l'utiliser à d'autres fins que celles initialement prévues.

\subsection{La création de tickers personnalisés}

On pourrait penser que les affordances prévues par les concepteurs ne contraignent pas discursivement les internautes puisqu'il est prévu des «tickers personnalisés ». J'en ai donc créé un pour une journée d'étude sur le thème des affordances qui a eu lieu à Montpellier le 28 juin 2017 : 


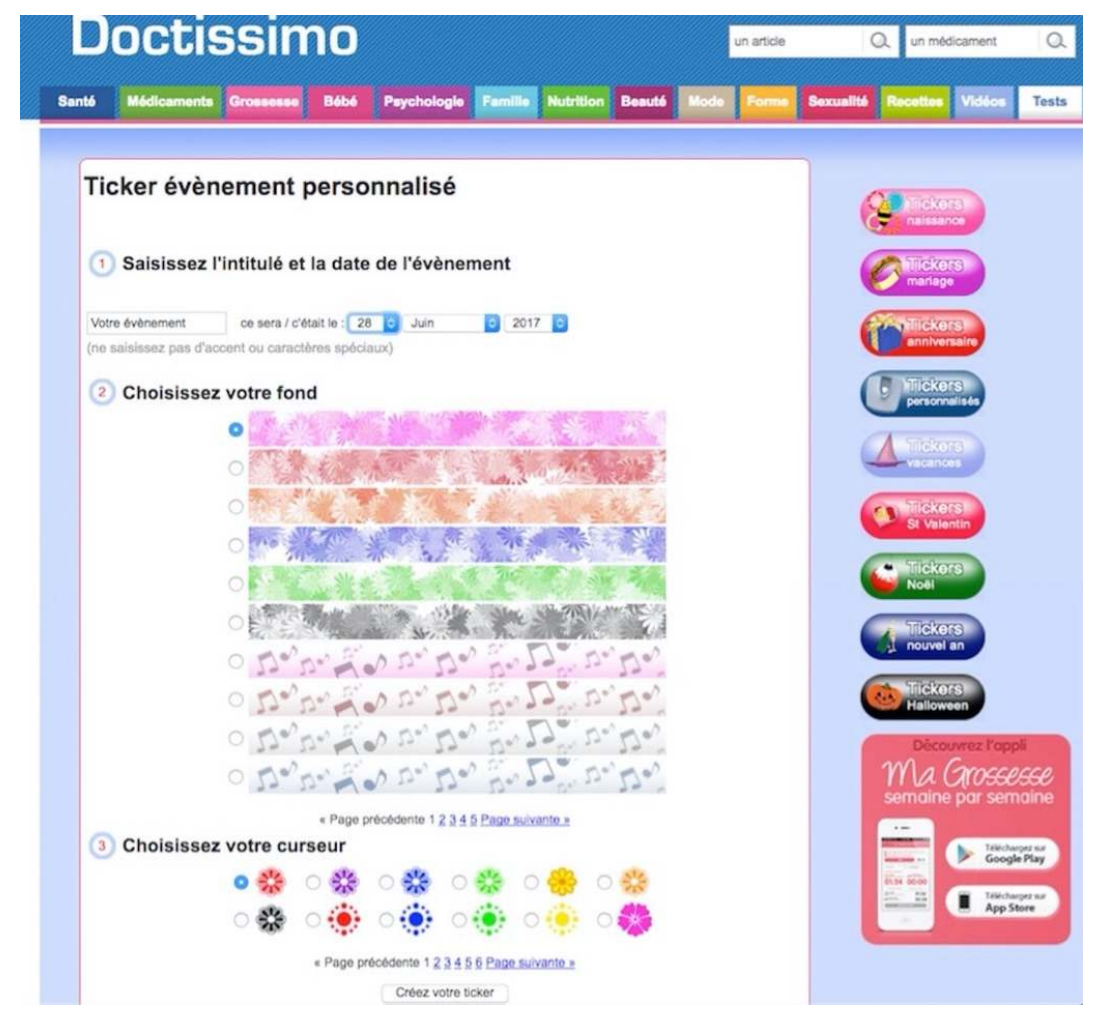

Fig. 9 : création d'un ticker personnalisé sur Doctissimo en 2017.

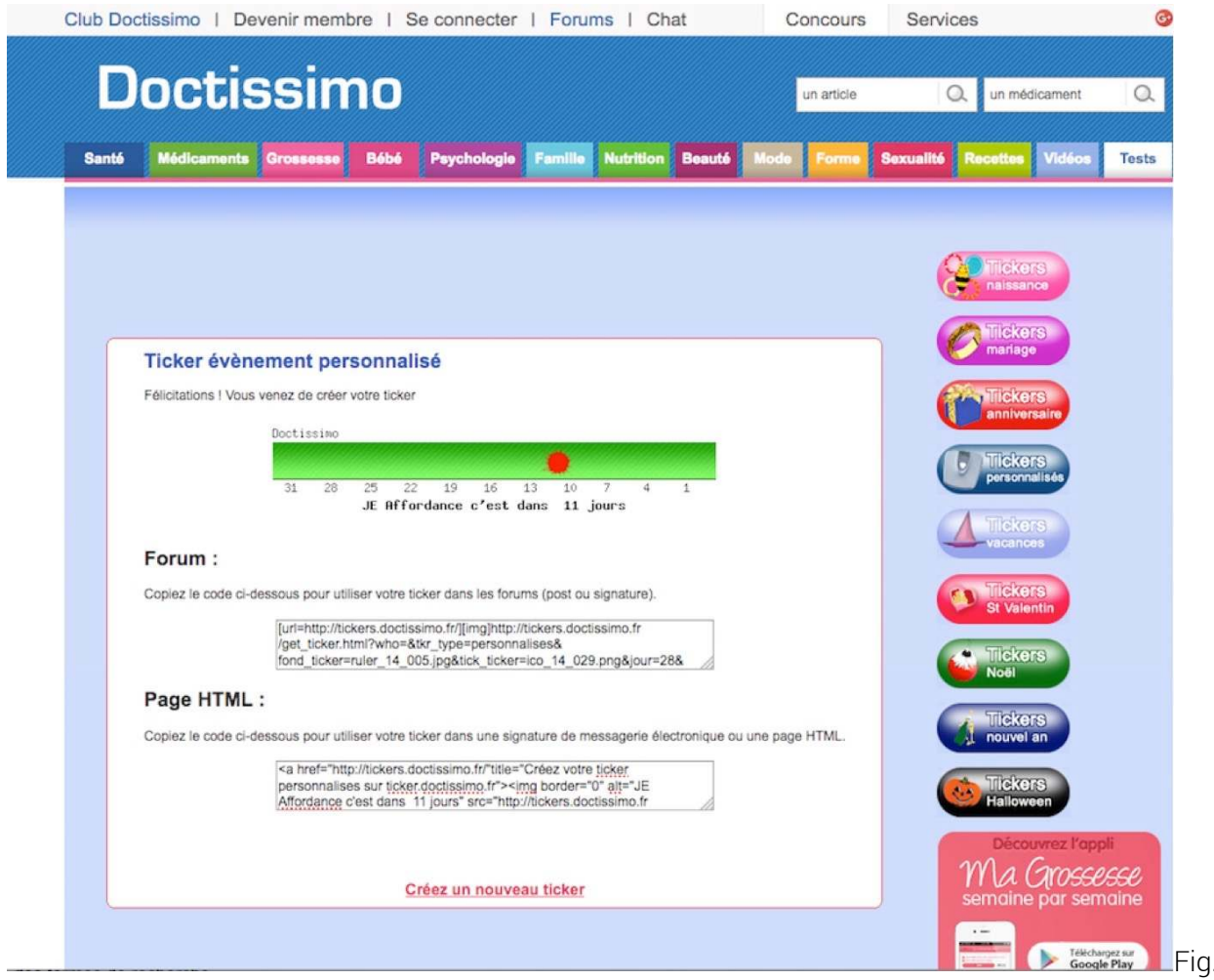

10 : affıchage du ticker personnalisé définitif sur Doctissimo (2017)

En figures 9 et 10, on constate que, même lorsqu'il s'agit de tickers personnalisés, tout au long de l'opération (choix de l'intitulé, de la date, du fond et du curseur), les modèles d'événements heureux me sont toujours proposés (bandeau à droite). Mon agir est donc très contraint par les suggestions et le dispositif qui impose texte et visuels. 


\subsection{Le détournement du ticker de vacances}

Pourtant, l'internaute ne s'appuie pas strictement sur ces modèles. Les images-perception affordent autre chose que la répétition textuelle.

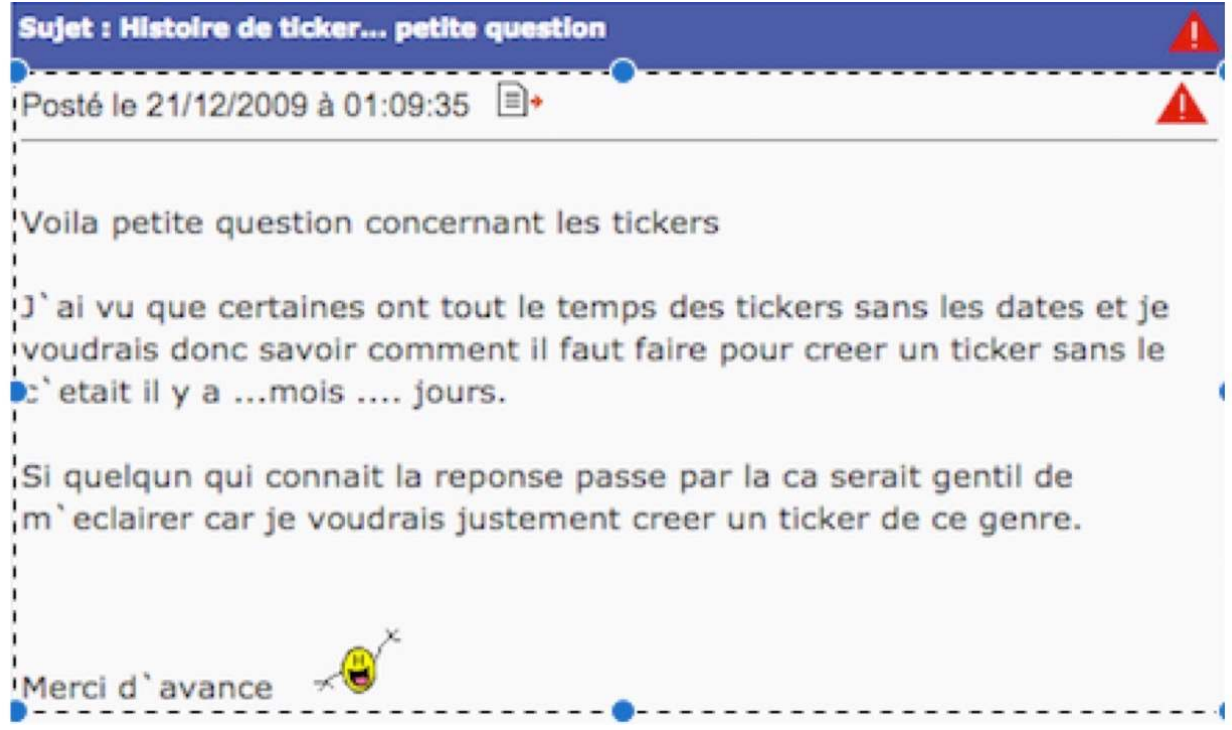

Fig. 11 : Question d'un internaute sur la création des tickers sur Doctissimo (2009).

\section{Posté le 21/12/2009 à 23:43:58}

en fait il faut faire un ticker vacances ca marche que avec les tickers vacances et mettre la date d'hier et hop plus de dates

\section{Jaime}

Fig. 12 : Réponse d'internaute sur la création d'un ticker sans dates sur Doctissimo (2009)

La figure 11 présente la question d'un internaute à la communauté : comment créer un ticker sans la précision temporelle "c'était il y a ...mois ...jours ». L'internaute fait référence aux discours préformés des tickers et souhaite se libérer du modèle textuel. En figure 12, un internaute donne la solution: « il faut faire un ticker vacances ça marche que avec les tickers vacances ». La restrictive que avec révèle que l'internaute a essayé les différents modèles. Il ne s'agit donc pas d'une affordance primaire. L'internaute a bel et bien cherché à détourner l'affordance prescrite du ticker. Il y est parvenu en utilisant :

1/ les modèles d'expérience (tickers vus chez d'autres internautes)

2/ la potentialité de l'objet ticker (en allant voir ce qu'il est possible de faire avec chaque ticker)

Cela a été conditionné par un besoin précis (non mentionné dans le corpus). Le deuil, catégorie n'appartenant pas aux événements heureux, est une des circonstances qui incitent les internautes à modifier le ticker. 


\subsection{Le détournement du ticker de naissance en ticker de deuil}

Le dispositif ne prévoit pas que des parents attendant un enfant puissent perdre leur enfant avant le terme, comme on peut le constater sur la figure ci-dessous :
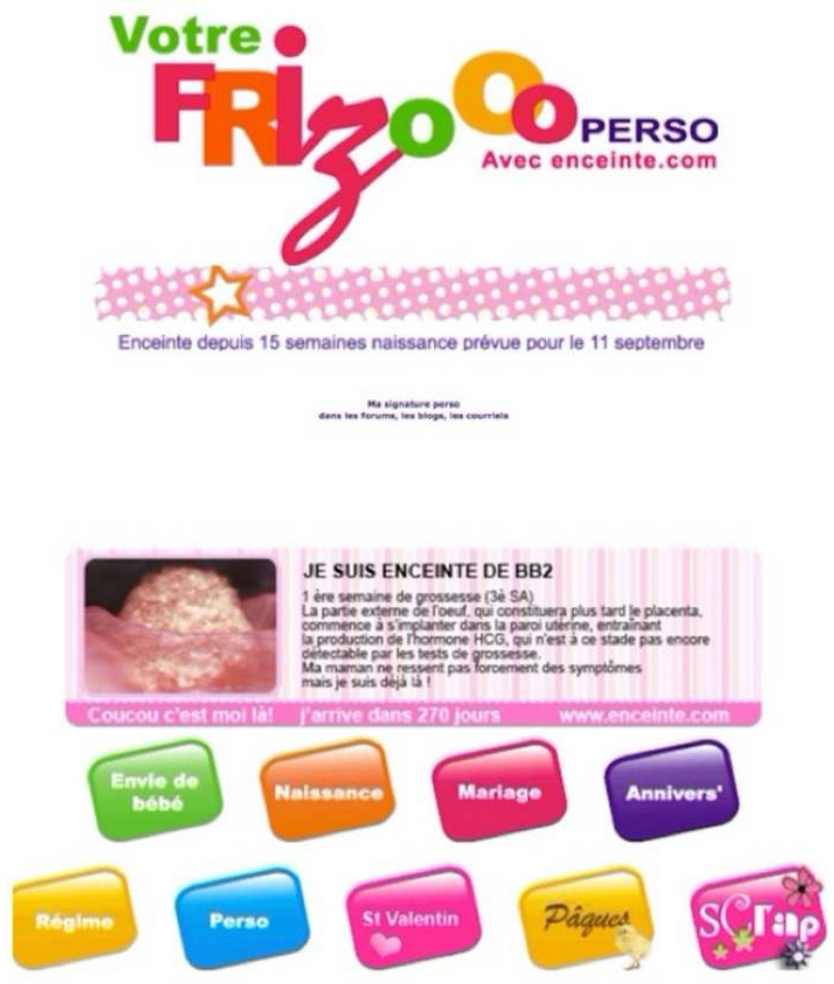

Fig. 13 : modèles de ticker sur le site enceinte.com en 2017.

Sur le site enceinte.com, il est prévu différents tickers, appelés ici frizooo: «Envie de bébé », " Naissance », " Mariage ", " Annivers ", " Régime ", "St Valentin », etc. Il est proposé d'indiquer les dates de début de grossesse et d'accouchement sur le mode de l'énonciation ventriloque (Paveau 2016) : Je suis enceinte de BB2, J'accouche dans 1 jour), avec un je plus vicaire que déictique qui permet à chacun de s'incarner dans le discours, mais qui montre bien que le concepteur parle à la place des concernées, les femmes enceintes, en leur imposant une vision du monde, celle d'un monde où les bébés ne meurent pas. 


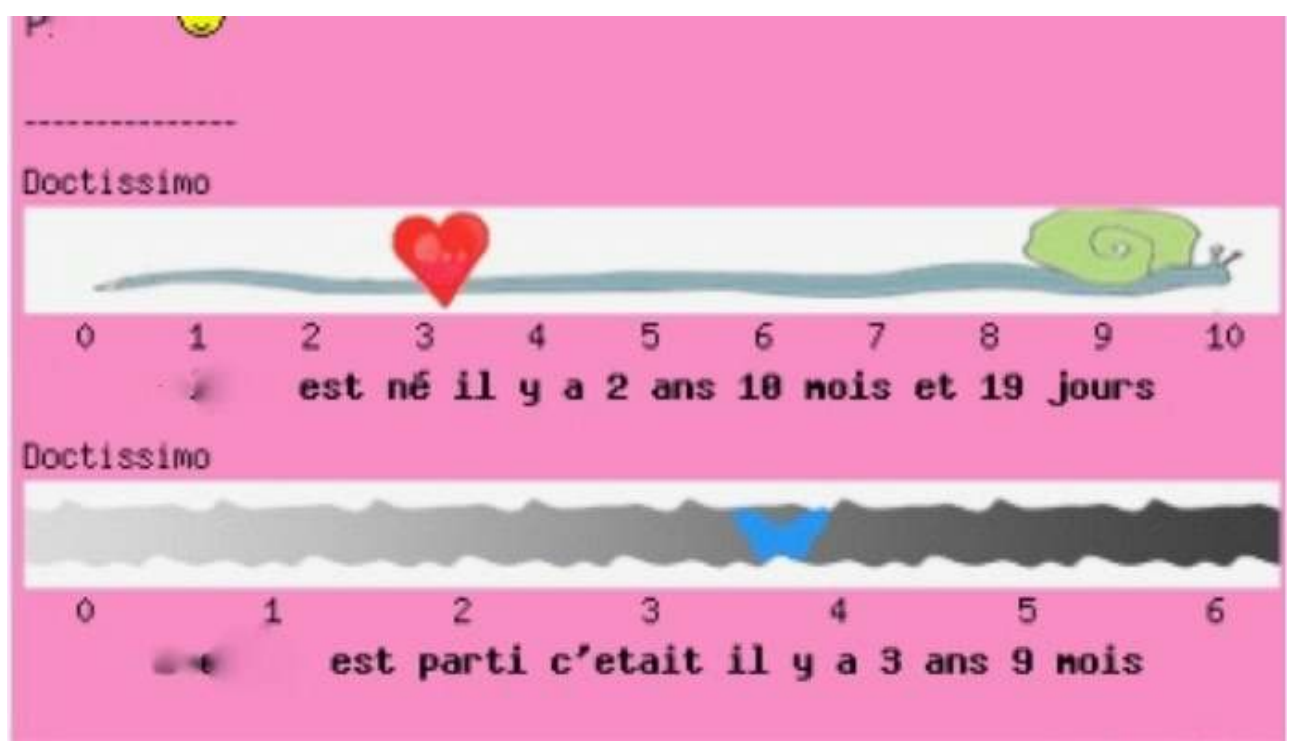

Fig. 14 : ticker de naissance et ticker épitaphier d'un enfant sur Doctissimo publiés en 2013.

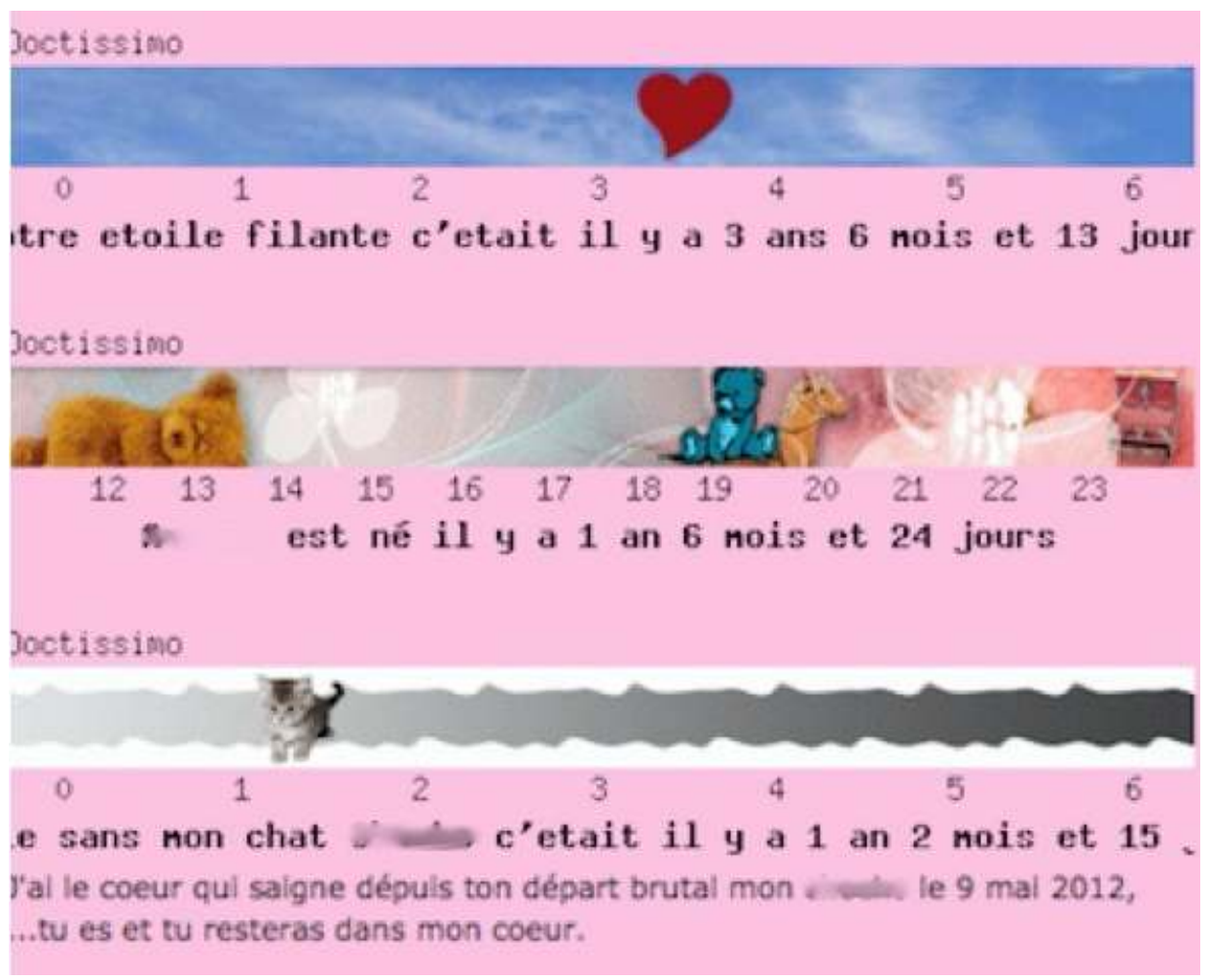

Fig. 15 : ticker de naissance et tickers épitaphiers (d'humain et d'animal) sur Doctissimo publiés en 2013.

Les internautes ayant perdu un enfant sont souvent familières des forums de grossesse et connaissent donc le principe du ticker. En gardant la même structure iconique, mais en adaptant le texte (remplacement de né par parti) comme sur la figure 14, l'internaute transforme le ticker de naissance en ticker de décès. Comme il est impossible de modifier le texte du ticker de naissance, l'internaute a dû recourir à un ticker personnalisé pour le bébé décédé mais a produit un énoncé construit sur le format d'un ticker de naissance (prénom + prédicat + référence temporelle). Cette ressemblance non fortuite prouve que c'est le ticker de naissance qui a affordé le ticker de décès, et non les «tickers 
personnalisés ». L'énonciatrice a choisi le seul modèle qui évoque le deuil (couleur grise) mais a repris un motif enfantin pour le curseur (papillon bleu). On voit qu'il en est de même en figure 15 pour le ticker épitaphier d'animal. A cet égard, cette note de Goffman dans son ouvrage Les cadres de l'expérience, est édifiante :

Mon intention n'est pas de dire que les objets fabriqués n'ont aucune signification stable, mais simplement que les circonstances peuvent leur en imposer une supplémentaire. Obus, jarres, déchets de plomberie peuvent être transformés en lampes de décoration; mais ce nouvel usage n'a de sens que si ces objets gardent leur sens premier. Le résultat n'est pas tant une lampe qu'une lampe originale. Le fait de subordonner un usage officiel à un usage irrévérencieux peut devenir un véritable sport, comme l'attestent les farceurs qui s'amusent à sonner aux portes de gens [...]. J'entends insister sur le fait que le sens d'un objet (ou d'un acte) est socialement défini et que cette définition tient au rôle de l'objet dans la société tout entière; ce rôle est ensuite appréhendé par des cercles plus limités comme un sens donné susceptible d'être modifié sans toutefois être totalement réinventé. Si le sens d'un objet tient évidemment à son usage, comme nous le rappelle le pragmatisme, il ne dépend pas de ses usages : tous les objets qui servent à enfoncer des clous ne sont pas des marteaux. (Goffman 1991 [1974] : 48)

61 Cette remarque peut être transposée au ticker dont l'usage socialement établi est celui d'une échelle temporelle associée à des événements heureux ou anodins (même l'arrêt de tabac, s'il n'est pas un événement « heureux », est un acte dont on se félicite). L'usage fait par le «cercle plus limité" des internautes endeuillées se superpose à cet usage socialement admis et met en relief une forme de tabou sur la mort. Les internautes savent à quoi réfère le cadre premier du ticker dans leur situation de mère (événement naissance).

62 Certes, à force de répétitions, l'acte de transformer un ticker de naissance en ticker de deuil devient routinier et le caractère inhabituel s'estompe peu à peu.

Le ticker de la figure 15 manifeste une double forme de détournement de la part de l'internaute: de même que le ticker n'a pas été conçu pour le deuil, il n'a pas été imaginé pour l'animal. On voit donc que l'usager outrepasse sur le plan des praxis idéologiques les fonctionnalités prévues par les concepteurs en adaptant les objets numériques afin de manifester une émotion particulière, celle associée à la perte d'un être cher (enfant ou animal). Plus encore, ce trajet de l'intentionnalité conduit au postspécisme : les tickers, déclencheurs d'empathie, sont employés aussi bien pour les humains que pour les animaux (les internautes mettent sur le même plan et une même page leurs enfants et leurs animaux).

\section{Conclusion}

64 Au niveau des affordances secondaires, le détournement de l'affordance injonctive par l' « usager » répond donc à l'intentionalité du concepteur. L'internaute est ainsi tout autant « concepteur » que « usager » puisqu'il élargit la gamme des tickers, devenant par ce geste un " produser » (Bruns 2007, 2008), c'est-à-dire un usager à la fois producteur et utilisateur. Ces usages « irrévérencieux » montrent que les internautes développent par l'usage une maîtrise de l'objet technologique. Quéré (1999) a révélé notamment que percevoir une affordance, c'est percevoir un contexte d'action : la maîtrise du signe n'est que le point d'entrée d'une maitrise beaucoup plus large. En s'appropriant un signe tel 
que le ticker, en dépassant le niveau de l'affordance prescrite, les internautes manifestent leur maittrise, ou du moins leur emprise sur la technologie, montrant qu'ils savent modaliser un cadre (en transposant l'univers du deuil à celui d'événements heureux) afin de manifester une émotion et de dire leur deuil.

Ces exemples de tickers épitaphiers montrent que la relation binaire concepteurutilisateur reflète une conception trop étroite des véritables usages. Les cas présentés incitent à considérer l'objet numérique de façon moins dualiste (où l'affordance fonctionnerait à sens unique, du concepteur vers l'utilisateur). Il reste à savoir si les concepteurs s'empareront à leur tour des « suggestions » des internautes, et s'il s'opèrera ainsi un mouvement inversé, les tickers créés par les internautes affordant la création de nouveaux modèles de tickers, comme ceux de deuil d'enfant ou d'animal. Par leurs tickers, les internautes bousculent les interdits du discours et remodèlent leur environnement numérique. Cette interactivité est d'ailleurs identifiable dans la présence récente de modèles de tickers reflétant un monde moins stéréotypé, avec par exemple l'ajout d'un modèle d'adoption sur Lilypie (ticker «adopting a baby » créé en 2004). On voit ainsi comment la dimension expressive est venu démultiplier les affordances d'objets numériques dont l'utilisation était suggérée, imposée, dans des cadres très contraints (naissance, mariage, etc.). Ce sont en partie les dispositions psychologiques des internautes en situation de deuil qui ont permis le détournement d'affordances.

\section{BIBLIOGRAPHIE}

Bradner Erin, 2001, Social Affordances of Computer-Mediated Communication Technology : Understanding Adoption, Seattle, CHI.

Bruns Axel, 2007, « Produsage : A Working Definition », Produsage.org. From Production to Produsage : Research into User-Led Content Creation [site], http://produsage.org/produsage, consulté le 26.08.2015.

Bruns Axel, 2008, Blogs, Wikipedia, Second Life, and Beyond. From Production to Produsage, New York, Peter Lang.

Costall Alan, 1982, On how so much Information Controls so much Behaviour : James Gibson's Theory of Direct Perception, in G. Butterworth (ed.), Infancy and Epistemology : an Evaluation of Piaget's Theory, New York, St Martin's Press : 30-51.

Franchak John M., van der Zalm Dina \& Adolph Karen E., 2010, « Learning by doing : Action performance facilitates affordance perception », Vision Research 50 : 2758-2765.

Gaisch Martina, 2015 [2014], Affordances for Teaching in an International Classroom, Sarrebruck, Südwestdeutscher Verlag für Hochschulschriften.

Genette Gérard, 1979, Introduction à l'architexte, Seuil.

Gibson James J. 1966, The Senses Considered as Perceptual Systems, Boston, Houghton Mifflin.

Gibson James J., 1977, «The Theory of Affordances », dans Shaw R. \& Brandsford J. (eds),

Perceiving, Acting, and Knowing : Toward and Ecological Psychology : 62-82. 
Gibson James J., 1979, The Ecological Approach to Visual Perception, Hillsdale, Lawrence Erlbaum.

Gibson James J. \& Crooks Laurence E., 1938, « A Theoretical Field-analysis of Automobiledriving », American Journal of Psychology 51 : 453-471.

Goffman Erving, 1991 [1974], Les Cadres de l'expérience, trad. Isaac Joseph, Paris, Les éditions de minuit.

Gülich Elizabeth et Krafft Ulrich, 1997, «Le Rôle du "préfabriqué” dans les processus de production discursive », in Michel Martins-Baltar (dir.), La Locution entre langues et usages, ENS Éditions : 241-276.

Kaufmann Laurence \& Clément Fabrice, 2007, « How Culture Comes to Mind : From Social Affordances to Cultural Analogie », Intellectica 46-47 : 221-250.

Jeanneret Yves et Souchier Emmanuël, 2005, « L'énonciation éditoriale dans les écrits d'écran », Communication \& langages $145: 3-15$.

Jones Keith S., 2003, « What Is an Affordance ?», Ecological Psychology, 15/2 : 107-114.

Lahlou Saadi, 2000, «Attracteurs cognitifs et travail de bureau », Intellectica 30 : 75-113.

Latour Bruno, 2007 [1994], « Une sociologie sans objet ? Remarques sur l'interobjectivité », dans Debary O., Objets et mémoires, Laval, MSH-Presses de l'Université Laval : 38-57.

Leńko-Szymańska Agnieszka \& Boulton Alex (eds), 2015, Multiple Affordances of Language Corpora for Data-driven Learning, Corpora 11, ATILF-CNRS.

Leroi-Gourhan André, 1945, Évolution et techniques, II. Milieu et techniques, Paris, Albin Michel. Lewin Kurt, 1926, «Vorsatz, Wille und Befürdnis mit Vorbemerkungen über die psychichen Kräfte und Energien und die Struktur der Seele », Psychologische Forschung 7 : 294-329. Lewin Kurt, 1968 [1938], The Conceptual Representation and the Measurement of Psychological Forces, Durham, Duke University Press.

Loyauté Benjamin, 2016, Le bruit des bonbons - The Astounding Eyes of Syria, Paris, Dilecta.

Loyauté Benjamin, 2017, Heterotopia, Affordance et Nouveau Pragmatisme, Paris, Dilecta.

Morgagni Simone, 2011, «Repenser la notion d'affordance dans ses dynamiques

sémiotiques ", Intellectica $55: 241-267$.

Niveleau Charles-Édouard, 2006, « Le concept gibsonien d'affordance : entre filiation, rupture et reconstruction conceptuelle », Intellectica : 159-199.

Norman Donald A., 1988, The psychology of everyday things, New York, Basic Books.

Norman Donald A., 2004, « Affordances and Design », [en ligne : https://www.researchgate.net/ publication/265618710_Affordances_and_Design].

Paveau Marie-Anne, 2012, «Ce que disent les objets. Sens, affordance, cognition », Synergies Pays riverains de la Baltique : 53-65.

Paveau Marie-Anne, 2016, "Parler du burkini sans les concernées. De l'énonciation ventriloque," dans La pensée du discours [carnet de recherche], https:// penseedudiscours.hypotheses.org/4734, consulté le 05 octobre 2016. 
Paveau Marie-Anne, 2018, « Pour une postlinguistique 4. Biographies de choses au-delà de l'humain », dans La pensée du discours [carnet de recherche], [en ligne : https:// penseedudiscours.hypotheses.org/15704], consulté le 27 juillet 2018.

Poché Fred, 2009, « La sémiosis et l'interprétation dans la "métaphysique scientifique" de Peirce ", Revue des sciences religieuses 4, [En ligne : http://rsr.revues.org/445] : 551-565.

Peirce Charles Sanders, 1978, Ecrits sur le signe rassemblés, traduits et commentés par Gérald Deledalle, Paris, Editions du seuil.

Quéré Louis, 1997, « La situation toujours négligée ?», Réseaux 85, [en ligne :

7971_1997_num_15_85_3139] : 163-192.

Quéré Louis, 1999, « Action située et perception du sens », dans M. Formel et L. Quéré (dir.), La Logique des situations. Nouveaux regards sur l'écologie des activités sociales, Paris, Éditions de l'EHESS : 300-338.

Ruchon Catherine, 2015, « Les maternités douloureuses dans les discours numériques », Communication \& langages $186: 111-127$.

von Uexküll Jakob, 2010 [1956/1934], Milieu animal et milieu humain, Paris, Payot \& Rivages, trad. Charles Martin-Freville, préface de Dominique Lestel.

Werner Heinz, 1957 [1926], Comparative Psychology of Mental Development [Einführung in die Entwicklungspsychologie], trad. E.B. Garside, New York, International Universities Press.

\section{NOTES}

1. C'est-à-dire que le trajet de la représentation parcourt une chaîne d'interprétants successifs : « [En bref, un signe est] tout ce qui détermine quelque chose d'autre (son interprétant) à renvoyer à un objet auquel lui-même renvoie (son objet) de la même manière, l'interprétant devenant à son tour un signe et ainsi de suite ad finitum. » (Peirce 1978 : 126). L'interprétant final, qui ne renvoie à aucun autre signe et que Peirce associe à l'habitude (1978: 130, 136-137), est celui qui clôt temporairement ce parcours de l'interprétation.

\section{RÉSUMÉS}

Dans cet article, il s'agira de questionner les affordances, autrement dit les « signes actanciels » (Uexküll 1934), les «valences» ou le «caractère de demande» (Werner 1926) d'objets numériques iconotextuels (Nerlich 1990) tels que l'échelle temporelle numérique dite ticker. Forme d'architexte (Genette 1979, Jeanneret et Souchier 2005), le ticker propose un modèle iconique et langagier qui simultanément incite à l'action tout en limitant ce champ d'action. La diversité d'affordances semble dépendre des potentialités intrinsèques à l'objet mais aussi de l'expérience de l'usager. Plus ce coefficient de diversité augmente, plus la possibilité de dépasser le niveau injonctif de l'affordance augmente. En dépit des affordances prévues par les concepteurs et de leurs intentionnalités, les usagers " détournent " les fonctionnalités d'objets numériques en conservant le design initial mais en modifiant les éléments textuels qui 
l'accompagnent, créant ainsi une nouvelle catégorie, plus invisible, de produsage (Bruns 2007, 2008).

In this article, we will question the affordances, in other words the "actancial signs" (Uexküll 1934), the "valences" or the "character of demand" (Werner 1926) of iconotextual digital objects (Nerlich 1990) such as the numerical time scale called ticker. Form of architext (Genette 1979, Jeanneret and Souchier 2005), the ticker proposes an iconic and linguistic model which simultaneously encourages the action while limiting this field of action. The diversity of affordances seems to depend on the intrinsic potentialities of the object but also on the user's experience. As this coefficient of diversity increases, so does the possibility of exceeding the injunctive level of affordance. Despite of the affordances expected by designers and their intentionality, users "divert" the functionalities of digital objects by retaining the original design but changing the textual elements that accompany it, creating a new, more invisible category of produsage (Bruns 2007, 2008).

\section{INDEX}

Mots-clés : affordance, iconotexte, intentionnalité, produsage, ticker.

Keywords : affordance, iconotext, intentionality, produsage, ticker

\section{AUTEUR}

\section{CATHERINE RUCHON}

Université Paul-Valéry Montpellier 3, Pléiade EA 7338 